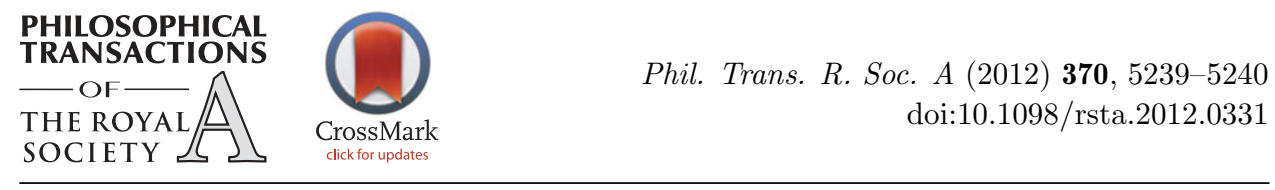

Preface

\title{
Principles and applications of quantum control engineering
}

This issue contains papers presented at the Theo Murphy Discussion Meeting Principles and applications of quantum control engineering held at the Kavli Royal Society International Centre, Chicheley Hall, 12-13 December 2011.

There has been a growing realization among scientists and engineers that we are witnessing the birth of a new industrial revolution based on quantum technologies. The main drivers for this have been the unrelenting trend of miniaturization, and the goal of exploiting quantum resources for computation and information processing. However, major advances have been made in areas such as nanotechnology, quantum chemistry, high-precision metrology, coherent opto-mechanics and electromagnetic circuits, etc., which have contributed to the growth of quantum engineering as a discipline deserving specific attention. To date, there have been many pioneering developments, both experimentally and theoretically, but it is fair to say that we are still at an early stage in the history of quantum technology, and that only time will tell which of several competing approaches and methodologies will emerge as the industry standards of the future.

Nevertheless, it has proved fruitful to look at the world of classical control engineering as a guide to future quantum technologies. Indeed, it now seems necessary to have a dedicated theory of quantum control, not just owing to the dynamical nature of quantum systems, but also to formulate the design problems specific to achieving the performance criteria desired of quantum systems. This meeting brought together several international experts working on quantum control, either as experimentalists who have directly applied control concepts, or as theoreticians who have developed modelling approaches for quantum control problems. The emphasis has been on the systematic development of quantum control as the engineering discipline that will guide emerging quantum technologies. The main applications covered, or suggested, include open-loop quantum control, adaptive feedback (quantum chemistry), real-time quantum feedback using measurement and filtering, and coherent control for closed-loop self-regulating system-controller models. The topics covered at the meeting include: rapid purification of quantum systems by measurement and control, controlling the light-matter interface, quantum filtering for systems driven by fields in non-classical input states, stabilization of quantum systems using quantum governors, pure Gaussian state and

One contribution of 15 to a Theo Murphy Meeting Issue 'Principles and applications of quantum control engineering'. 
entanglement generation; characterization and estimation of quantum systems; quantum systems identification, model reduction and synthesis with quantum feedback networks and quantum-coherent feedback in photonic circuits.

In addition to the proceedings contained in this issue, each of the talks concluded with an extended discussion session and these are available as audio downloads from the webpage: http://royalsociety.org/events/2011/quantumcontrol-engineering/

The organizing committee thank all those who participated in and contributed to the meeting, and to the Kavli Royal Society International Centre for kind support in hosting this event.

John E. Gough

Institute of Mathematics and Physics, Aberystwyth University, Aberystwyth, Ceredigion SY23 3BZ, UK E-mail address: jug@aber.ac.uk 